\title{
Nanotechnological Contribution to Drug Delivery System: A Reappraisal
}

\author{
Pramod Vishwanath Prasad1*, Tulsidas G. Shrivastav ${ }^{2}$ \\ ${ }^{1}$ Center for Biomedical Research, The Population Council, The Rockefeller University, New York, NY 10065, USA \\ ${ }^{2}$ Department of Reproductive Biomedicine, National Institute of Health and Family Welfare, New Delhi, India \\ Email: drpramod.pusa@gmail.com, ${ }^{*}$ pkbiochem@yahoo.com
}

Received 7 June 2014; revised 5 July 2014; accepted 21 July 2014

Copyright (C) 2014 by authors and Scientific Research Publishing Inc.

This work is licensed under the Creative Commons Attribution International License (CC BY). http://creativecommons.org/licenses/by/4.0/

(c) (i) Open Access

\begin{abstract}
With the emergence of Nanotechnology, there has been remarkable improvement in the field of drug delivery system over the past decade. Nanotechnology in drug delivery system is an upcoming field and significant research has been conducted in this regard. It has been able to overcome some limitations encountered with the traditional routine drug delivery systems and hence emerged as an effective alternative. This article reviews nanotechnology based different types of drug delivery systems and their therapeutic applications.
\end{abstract}

\section{Keywords}

Nanomedicine, Nanotechnology, Drug Delivery System, Application

\section{Introduction}

Nanotechnology is a branch of science which involves creating materials, devices or systems on a nanometer scale. It can be defined as the technology at the scale of one-billionth of a meter. It is the design, characterization, synthesis and application of materials, structures, devices and systems by controlling the shape and size at nanometer scale [1]-[3]. It is an interdisciplinary field and has three main extensively overlapping areas which are nanoelectronics, nanomaterials and nanobiotechnology. The prefix "nano" was first added to the word "technology" by Nario Taniguchi in 1974. He used the composite word to signify machining with tolerances of less than one micron [4]. Nanotechnology is most appropriately called nanoscience which refers to research at the scale of $100 \mathrm{~nm}$ or less. One of the most important areas in nanotechnology is nanomedicine referring to highly specific medical intervention at the molecular scale for diagnosis, prevention and treatment of diseases. Drug delivery nanosystems comprise a significant component of nanomedicine. Many of the current "nano” drug de-

*Corresponding author. 
livery systems are remnants of conventional drug delivery systems which are at the nanometer range [5]. With the burgeoning applications of "nanotechnology" in the health care and pharmaceutical industries it has been estimated that $80 \%$ of the market will be related to nanotechnology by 2015 [6].

The pharmaceutical companies are striving to provide the right therapy with minimal side effects at a reduced cost. Nanoparticles have the potential of improving the common drug delivery systems like oral, parenteral, ocular etc. and hence play a promising role in drug delivery systems. Some of the challenges of drug delivery systems include poor bioavailability, reduced therapeutic effectiveness, side effects and fluctuations in the drug-plasma concentrations. Nanotechnology in drug delivery systems is used to overcome these shortcomings [3]. Because the superiority of nanoparticles used in drug delivery system over the conventional drug delivery system lies in the following facts: 1) They have high surface area to volume ratio (due to very small size). Therefore, they exhibit improved loading and releasing efficiency of drugs [7]; 2) They improve bioavailability and circulation-time of drug as they can bypass the liver and thus preventing first pass metabolism [8] [9]; 3) They fascilitate localized, controlled, targeted (at specific sites, intracellular) delivery of drugs. Thus, they significantly lower the dose of drug (i.e., overall drug consumption) and side effects (toxicity). Moreover, through the manipulation of the characteristics of polymers, release of drug from nanostructures can be controlled to achieve the desired therapeutic concentration for the desired duration [10]; 4) They enhance solubility of drugs exhibiting poor solubility in water by using micelles (made up of amphiphilic materials). Thus, allow for the delivery of all types of drugs; 5) They have ability to cross biological membranes; and 6) They allow one to change pharmacokinetic properties of drug without changing the active compound [11]-[14]. Therefore, nanotechnology has become an important field which when applied in drug delivery systems can expand drug markets.

Most of the current research focuses on utilizing nanoparticles as drug delivery vehicles in most chronic diseases like cancer, HIV and diabetes where treatment options are limited. Generally, nanoparticles are in the form of polymers, ceramics, metals and biological materials with various forms. They might have spherical, branched or shell structures. Each structure offers unique characteristics that make it a suitable drug delivery system for a particular therapy [15] [16]. In the present review, we emphasize on some of the important nanoparticles which are employed as drug delivery systems.

\section{Nanoparticles as Drug Delivery Systems}

\section{(a) Nanoemulsions}

Emulsion is defined as the even distribution of one liquid into another. This property of mixing of two liquids has been used in delivery of materials in drugs and cosmetics. Nanoemulsions are ultra fine emulsions with droplet size below $1 \mu$. They are transparent in nature due to the minute size and have good biophysical and sensorial properties. Nanoemulsions are biocompatible and biodegradable. They can be produced easily and used as carriers for lipophilic drugs which are prone to hydrolysis [17]. They enhance gastrointestinal absorption and reduce inter- and intra-subject variability for various drugs. Due to their very large interfacial area, they exhibit excellent drug release profile [18]. However, they have been found to be metastable and may be destabilized due to Ostwald ripening. To avoid these problems, a number of factors have to be considered during the synthesis process. These factors include selecting an appropriate composition, controlling the order of addition of components, applying the shear in a manner that will effectively rupture the droplets, and ensuring that the dispersed phase molecules are insoluble in the continuous phase so that Ostwald ripening does not occur rapidly [19].

\section{(b) Liposomes}

Liposomes are vesicular structures with an aqueous core surrounded by a hydrophobic lipid bilayer, created by the extrusion of phospholipids [20]. They may be single layered or multi layered, varying in size from $15 \mathrm{~nm}$ to $100 \mathrm{~nm}$ or more. They have been found to be useful in cosmetics, drug delivery and food industries. Liposomes are used as drug carriers due to their ability to prevent degradation of drugs, reduce side effects and target drugs to site of action [21]. Liposomes work by attaching to the target cell or by its engulfment into the target cell, followed by the release of the drug encapsulated within to perform its function. For the attachment or engulfment to take place the modification of the surface properties of the liposome is essential. Surface modification is carried out based on the application of the liposome. They are used in transdermal drug delivery to potentiate skin permeation of drugs with high molecular weight and poor water solubility [22]. Liposomes have been used as carriers for gentamicin in order to reduce toxicity, as a means of delivery of drugs to the lungs by nebulization and in ocular delivery [23] [24]. 


\section{(c) Transferosomes}

Transferosomes are a modified form of liposomes. They are more elastic than liposomes which make them a good vessel for transdermal drug delivery by deforming and passing through even narrow constrictions. The flexibility can be altered by changing the composition and mixing suitable surface active agents [25]. Transferosomes possess an infrastructure consisting of hydrophobic and hydrophilic moieties together and as a result can accommodate drug molecules with wide range of solubility [26]. They are able to entrap a variety of drugs of varying molecular weights. They prevent the degradation of drugs and can be used for controlled drug delivery over a long period of time. Transferosomes also have their drawbacks like high cost, predisposition to oxidative degradation, chemical instability and concern regarding the purity of the phospholipids used [26].

\section{(d) Dendrimers}

Dendrimers are unimolecular, monodisperse, micellar nanostructures, approximately $20 \mathrm{~nm}$ in size. They are globular having a central symmetric core from which arise highly branched structures. Drugs can be encapsulated within the core or attached to the surface [27]. Dendrimers provide controlled release from the inner core and the encapsulation of both hydrophobic and hydrophilic drugs [28]. Since they have multiple attachment sites a number of different types of molecules such as drugs, solubilizing groups, etc. can be attached to the surface in a defined manner as required. In this way, the surface can be modified according to the desired application.

Dendrimers have been extensively studied in the delivery of antitumor, antiviral, and antibacterial drugs, in intracellular drug delivery and gene delivery. They are also used in photodynamic therapy to detect tumors [29]. Variation in toxicity has been noted with the change in the functional groups attached. For example, it has been studied that primary amines are more toxic than secondary or tertiary amines [30]. The material of the core also affects the toxicity of the dendrimers. It is suggested that the aromatic interior of the dendrimer may cause hemolysis through hydrophobic membrane contact [31].

\section{(e) Ceramic Nanoparticles}

Particles such as alumina, silica and titania are called as ceramic nanoparticles. These are porous, inorganic compounds which are biocompatible in nature. Because these particles can be easily engineered with the desired size and porosity, growing interest has recently emerged to utilize ceramic nanoparticles as drug vehicles [32]. These particles protect doped molecules (enzymes, drugs, etc.) against denaturation induced by external $\mathrm{pH}$ and temperature. Their ultra-low size (less than $50 \mathrm{~nm}$ ) can help them evade the reticulo-endothelial system (RES) of the body [33]. Ceramic (inorganic) particles have been highlighted to provide increased mechanical strength, chemical stability, biocompatibility, and resistance to microbial attack as compared to their organic (polymeric) counterparts [33]. Ceramic nanoparticles have been used in research involving cancer therapy such as targeting cancerous cells in liver as well as in the delivery of insulin by oral administration. They are also effective in delivering proteins and genes. However, these particles are not biodegradable and so there is a concern that they may accumulate in the body and cause harmful effects [34].

\section{(f) Lipid Nanocarriers}

Lipid nanocarriers, as the name suggests are nanoparticles with a lipid matrix. The different lipid based carriers include solid lipid nanoparticles (SLN), nanostructured lipid carriers (NLC) and lipid drug conjugate (LDC). These contain lipids, drugs and surfactants which act as stabilizers. The first generation of solid lipid nanoparticles (SLN) was developed at the beginning of the nineties as an alternative carrier system to emulsions, liposomes and polymeric nanoparticles. The main features of SLN with regard to parenteral application are the excellent physical stability, protection of incorporated labile drugs from degradation, controlled drug release (fast or sustained) depending on the incorporation model, good tolerability and site specific targeting [35]. However, SLN have demerits such as high water content, drug expulsion after polymorphic transition on storage and insufficient loading capacity limited by the solubility of drug in lipid melt.

Nanostructured lipid carriers which are also solid at room temperature are produced by the blend of a solid lipid with a liquid lipid. They were introduced to overcome the limitations of SLN. They show an increase in the payload and prevent drug expulsion. NLC have been used mainly for topical delivery and hence find application in cosmetics and dermatology [35].

Lipophilic drugs are usually incorporated in SLN but due to partitioning effects during production, only highly potent hydrophilic drugs effective in low concentrations are incorporated in SLN. LDC allows the incorporation of hydrophilic as well as lipophilic drugs and has a drug loading capacity of up to 33\% [35].

(g) Polymeric Nanoparticles

Polymeric nanoparticles are solid, colloidal particles which may have varying shapes such as spherical, 
branched or shell structures. They vary in size from $10 \mathrm{~nm}$ to $1000 \mathrm{~nm}$. Their main advantage is their size which enables them to penetrate capillaries [15]. Drugs can be introduced into them by encapsulation, attachment and entrapment enabling a sustained drug release system. The extent of uptake by cells depends on the type of polymer used. Polymeric nanoparticles have been used for vaccines and cancer chemotherapeutics for more than 45 years. Some other applications of these nanoparticles include possible recognition of vascular endothelial dysfunction; oral delivery of insulin, brain drug targeting for neurodegenerative disorders such as Alzheimer's disease, topical administration to enhance penetration and distribution in and across the skin barrier, and application as $\mathrm{pH}$-sensitive nanoparticles to improve oral bioavailability of drugs such as cyclosporine A [32]. However, the main concern of polymeric nanoparticles is the possible cytotoxic effect which may prove to be a major drawback.

\section{(h) Other Drug Delivery Systems}

Ethosomes are similar to liposomes but have high alcohol content. The ethanol from ethosomes' composition plays the same role as the surfactant from the transferosomes, namely disorganizing the lipid bilayer and thus conferring a ten times higher deformability to the particles [36] [37].

Polymeric micelles are composed of amphiphilic block copolymers. They have a hydrophobic inner core surrounded by a shell of hydrophilic polymers. They are able to reach targets that are not accessible by liposomes, but have a limited targeting ability due to the low drug loading and low drug incorporation stability.

Nanocapsules are hollow structures having a polymer membrane which encapsulates the drug in the cavity. Metallic nanoparticles, as the name suggests are made up of metals such as iron oxide, gold, silver and nickel. They are used for targeted drug delivery wherein their surface can be modified by changing the functional groups according to the application. Carbon nanomaterials include carbon nanotubes and fullerenes. They have been studied for various therapeutic applications; however, the toxicity of carbon nanotubes cannot be ignored.

\section{Conclusions}

Some of the common challenges for most drug delivery systems are poor bioavailability, intestinal absorption, sustained and targeted delivery to the site of action, therapeutic effectiveness, adverse effects and fluctuation in the drug-plasma concentration. Incorporating Nanotechnology in the drug delivery system is a step forward to overcome all these challenges. A better understanding and application of nanotechnology in drug delivery systems can play a significant role in improving the efficacy of treatment, patient compliance and make the treatment cost effective for the patients.

Possible hazards of nanoparticles, however, to both health and environment cannot be ignored. Most of these risks are due to the high surface area to volume ratio (due to very small size) which might make the particles very catalytic or reactive [38]-[40]. Moreover, their interactions with biological systems need to be explored as they can pass through cell membranes [41].

\section{Acknowledgements}

We are very much thankful to the anonymous reviewers for providing valuable comments on this review article. They have tremendously helped us to improve the quality of our representation.

\section{References}

[1] The Royal Society and the Royal Academy of Engineering (2014) Nanoscience and Nanotechnologies. The Royal Society and the Royal Academy of Engineering Report. http:/www.nanotec.org.uk/finalReport.htm

[2] Stylios, G.K., Giannoudis, P.V. and Wan, T. (2005) Applications of Nanotechnologies in Medical Practice. Injury, 36, S6-S13.

[3] Nelson, A.O., Patrick, O.O. and Ndidi, C.N. (2009) Nanotechnology and Drug Delivery. Part 1: Background and Applications. Tropical Journal of Pharmaceutical Research, 8, 265-274.

[4] Taniguchi, N. (1974) Proc Int Conf Prod Eng, Part II, Japan Society of Precision Engineering, Tokyo.

[5] Nanotechnology, K.P. (2007) What It Can Do for Drug Delivery. Journal of Controlled Release, 120, 1-3. http://dx.doi.org/10.1016/j.jconrel.2007.05.003

[6] The Nanotechnology Opportunity Report 2008. 3rd Edition. www.cientifica.com

[7] Panyam, J. and Labhasetwar, V. (2003) Biogradebale Nanoparticle for Drug and Gene Delivery to Cells and Tissue. 
Advanced Drug Delivery Reviews, 55, 329-347. http://dx.doi.org/10.1016/S0169-409X(02)00228-4

[8] Italia, J.L., Bhatt, D.K., Bhardwaj, V., Tikoo, K. and Ravi, K. (2007) PLGA Nanoparticle for Oral Delivery of Cyclosporine: Nephrotoxicity and Pharmacokinetic Studies in Comparison to Sandimmune Neural. Journal of Controlled Release, 119, 197-206. http://dx.doi.org/10.1016/j.jconrel.2007.02.004

[9] Sahoo, S.K. and Labhasetwar, V. (2003) Nanotech Approaches to Drug Delivery and Imaging. Drug Discovery Today, 8, 1112-1120. http://dx.doi.org/10.1016/S1359-6446(03)02903-9

[10] Peppas, N.A. (2004) Intellegent Therapeutics: Biomimetic Systems and Nanotechnology in Drug Delivery. Advanced Drug Delivery Reviews, 56, 1529-1531. http://dx.doi.org/10.1016/j.addr.2004.07.001

[11] University of Waterloo (2010) Nanotechnology in Targeted Cancer Therapy. http://www.youtube.com/watch?v=RBjWwlnq3cA

[12] Lavan, D.A., McGuire, T. and Langer, R. (2003) Small Scale Systems for in Vivo Drug Delivery. Nature Biotechnology, 21, 1184-1191. http://dx.doi.org/10.1038/nbt876

[13] Cavalcanti, A., Shirinzadeh, B., Freitas Jr., R.A. and Hogg, T. (2008) Nanorobot Architecture for Medical Target Identification. Nanotechnology, 19, Article ID: 015103. http://dx.doi.org/10.1088/0957-4484/19/01/015103

[14] Gonsalves, K.E., et al. (2008) Biomedical Nanostructures. John Wiley \& Sons, Inc., Hoboken, New York, USA.

[15] Brigger, I., Dubernet, C. and Couvreur, P. (2002) Nanoparticles in Cancer Therapy and Diagnosis. Advanced Drug Delivery Reviews, 54, 631-651. http://dx.doi.org/10.1016/S0169-409X(02)00044-3

[16] Prasad, P.V., Kumar, A., Pal, P.C., Sharma, R.S., Rao, D.N., Shrivastav, T.G. and Ge, R.-S. (2011) Bird’s Eye View on the Recent Advances in Drug Delivery Systems. Journal of Biomaterials and Nanobiotechnology, 2, 544-556. http://dx.doi.org/10.4236/jbnb.2011.225065

[17] Santos-Magalhaes, N.S., Pontes, A., Pereira, V.M.W. and Caetano, M.N.P. (2000) Colloidal Carriers for Benzathin Penicillin G: Nanoemulsion and Nanocapsules. International Journal of Pharmaceutics, 208, 71-80. http://dx.doi.org/10.1016/S0378-5173(00)00546-9

[18] Brusewitz, C., Schendler, A., Funke, A., Wagner, T. and Lipp, R. (2007) Novel Poloxamer-Based Nanoemulsions to Enhance the Intestinal Absorption of Active Compunds. International Journal of Pharmaceutics, 329, 1173-1181. http://dx.doi.org/10.1016/j.ijpharm.2006.08.022

[19] Chiesa, M., Garg, J., Kang, Y.T. and Cheng, G. (2008) Thermal Conductivity and Viscosity of Water-in-Oil Nanoemulsions. Colloids and Surfaces A: Physicochemical and Engineering Aspects, 326, 67-72.

[20] Anju, S., Senthil, K. and Mahadevan, N. (2012) Nanotechnology: A Promising Approach for Cosmetics. International Journal of Advances in Pharmaceutical Research, 2, 54-61.

[21] Soppimath, K.S., Aminbhavi, T.M., Kulkarni, A.R. and Rudzinski, W.E. (2001) Biogradedable Polymeric Nanoparticles as Drug Delivery Devices. Journal of Recent Controlled Release, 70, 1-20. http://dx.doi.org/10.1016/S0168-3659(00)00339-4

[22] Qiu, Y., Gao, Y., Hu, K. and Li, F. (2008) Enhancement of Skin Permeation of Docetaxel: A Novel Approach Combining Micro Needle and Elastic Liposomes. Journal of Controlled Release, 129, 144-150. http://dx.doi.org/10.1016/j.jconrel.2008.04.019

[23] Jia, Y., Joly, H. and Omri, H. (2008) Liposomes as a Carrier for Gentamicin Delivery: Development and Evaluation of the Physiochemical Properties. International Journal of Pharmaceutics, 359, 254-263. http://dx.doi.org/10.1016/j.ijpharm.2008.03.035

[24] Zaru, M., Mourtas, S., Klepetsanis, P., Fadda, A.M. and Antimisiaris, S.G. (2007) Liposomes for Drug Delivery to the Lungs by Nebulization. European Journal of Pharmaceutics and Biopharmaceutics, 76, 655-666. http://dx.doi.org/10.1016/j.ejpb.2007.04.005

[25] Kulkarni, P.R., Yadav, J.D., Vaidya, K.A. and Gandhi, P.P. (2011) Transferosomes: An Emerging Tool for Transdermal Drug Delivery. IJPSR, 2, 735-741.

[26] Pandey, S., Goyani, M., Devmurari, V. and Fakir, J. (2009) Transferosomes: A Novel Approach for Transdermal Drug Delivery. Der Pharmacia Lettre, 1, 143-150.

[27] Svenson, S. and Tomalia, D.A. (2005) Dendrimers in Biomedical Applications-Reflections on the Field. Advanced Drug Delivery Review, 57, 2106-2129. http://dx.doi.org/10.1016/j.addr.2005.09.018

[28] Nelson, A.O., Patrick, O.O. and Ndidi, C.N. (2009) Nanotechnology and Drug Delivery. Part 2: Nanostructures for Drug Delivery. Tropical Journal of Pharmaceutical Research, 8, 275-287.

[29] Boas, U. and Heegaard, P.M.H. (2004) Dendrimers in Drug Research. Chemical Society Reviews, 33, 43-63. http://dx.doi.org/10.1039/b309043b

[30] Fischer, D., Li, Y.X., Ahlemeyer, J., Krieglstein, J. and Kissel, T. (2004) In Vitro Cytotoxicity Testing of Polycations: 
Influence of Polymer Structure on Cell Viability and Hemolysis. Biomaterials, 24, 1121-1131. http://dx.doi.org/10.1016/S0142-9612(02)00445-3

[31] Malik, N., Wiwattananpatapee, R., Klopsche, R., Lorenz, K., Frey, H., Weener, J.W., Meijer, E.W., Paulus, W. and Duncan, R. (2000) Dendimers: Relationship between Structure and Biocompatibility in Vitro, and Preliminary Studies on the Biodistribution of ${ }^{125}$ I-Labelled Polyamidoamine Dendrimers in Vivo. Journal of Controlled Release, 65, 133148. http://dx.doi.org/10.1016/S0168-3659(99)00246-1

[32] Yih, T.C. and Al-Fandi, M. (2006) Engineered Nanoparticles as Precise Drug Delivery Systems. Journal of Cellular Biochemistry, 97, 1184-1190. http://dx.doi.org/10.1002/jcb.20796

[33] Jain, T.K., Roy, I., De, T.K. and Maitra, A. (1998) Nanometer Silica Particles Encapsulating Active Compunds: A Novel Ceramic Drug Carrier. Journal of the American Chemical Society, 120, 11092-11095. http://dx.doi.org/10.1021/ja973849x

[34] Medina, C., Santos, M.J., Radomski, A., Corrigan, O. and Radomski, M.W. (2007) Nanoparticles: Pharmacological and Toxicological Significance. British Journal of Pharmacology, 150, 552-558.

[35] Wissing, S.A., Kayser, O. and Muller, R.H. (2004) Solid Lipid Nanoparticles for Parenteral Drug Delivery. Advanced Drug Delivery Reviews, 56, 1257-1272. http://dx.doi.org/10.1016/j.addr.2003.12.002

[36] Godin, B. and Touitou, E. (2003) Ethosomes: New Prospects in Transdermal Delivery. Critical Reviews in Therapeutic Drug Carrier Systems, 20, 63-102.

[37] Jain, S., Umamaheshwari, R.B., Bhadra, D. and Jain, N.K. (2004) Ethosomes: A Novel Vesicular Carrier for Enhanced Transdermal Delivery of an Anti-HIV Agent. Indian Journal of Pharmaceutical Sciences, 66, 72-81.

[38] Mnyusiwalla, A., Daar, A.S. and Singer, P.A. (2003) Mind the Gap: Science and Ethics in Nanotechnology. Nanotechnology, 14, R9-R13. http://dx.doi.org/10.1088/0957-4484/14/3/201

[39] Basi, C. (2013) Toxic Nanoparticles Might Be Entering Human Food Supply. MU Study Finds, University of Missouri, Columbia.

[40] Jackie, Y. (2001) Nanostructured Materials. Academic Press, New York.

[41] Nanotechnologies: 6. What Are Potential Harmful Effects of Nanoparticles? (ec.europa.eu) 
Scientific Research Publishing (SCIRP) is one of the largest Open Access journal publishers. It is currently publishing more than 200 open access, online, peer-reviewed journals covering a wide range of academic disciplines. SCIRP serves the worldwide academic communities and contributes to the progress and application of science with its publication.

Other selected journals from SCIRP are listed as below. Submit your manuscript to us via either submit@scirp.org or Online Submission Portal.
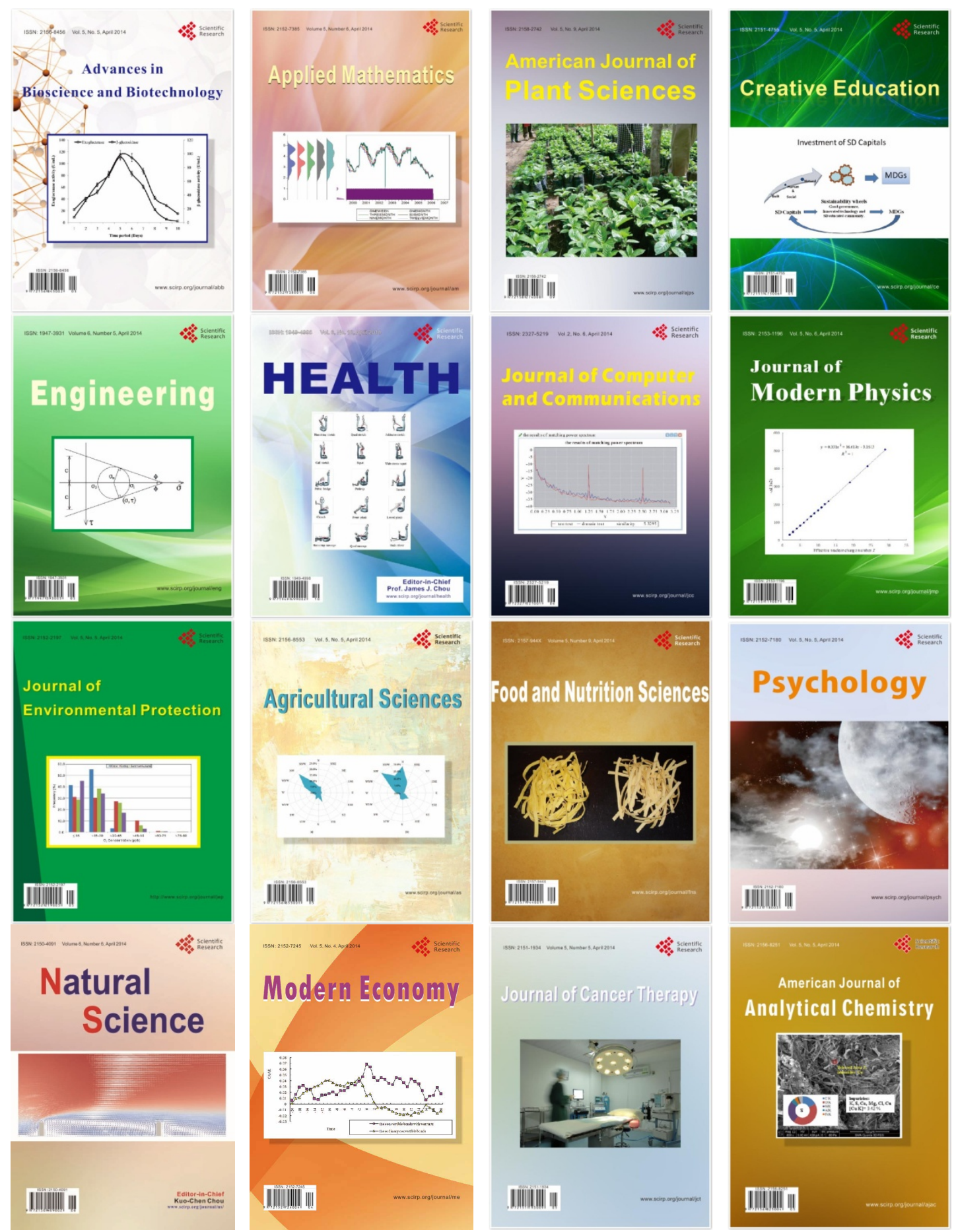